\title{
Enfermedad por modelantes asociado a cáncer de mama
}

\author{
Modeling disease associated with breast cancer \\ Jocelyn Pumares Campos, ${ }^{*}$ Luis Antonio Estrada Salazar, * \\ Ángela Hernández Rubio, ${ }^{\ddagger}$ Xicoténcatl Jiménez Villanueva ${ }^{\S}$
}

\author{
* Médico Residente. \\ ₹ Profesor adjunto. \\ $\S$ Profesor Titular del Curso \\ de Ginecología Oncológica. \\ División del Servicio de \\ Oncología del Hospital Juárez de \\ México, Facultad de Medicina \\ de la Universidad Nacional \\ Autónoma de México. México.
}

\begin{abstract}
RESUMEN
La alogenosis iatrogénica o enfermedad por modelantes es producida por el uso indiscriminado de sustancias alogénicas o exógenas. Es un procedimiento usado desde el siglo XIX con fines estéticos para mejorar el contorno corporal. En México, es una práctica frecuente; en la actualidad, representa un serio problema de salud pública. El desconocimiento de la fisiopatología de la alogenosis iatrogénica y la falta de información de los pacientes y del personal médico lleva a complicaciones que empeoran o agudizan un cuadro de características inmunológicas silentes. El espectro de la enfermedad, en su presentación clínica tanto local como sistémica, es consecuencia directa de la activación del sistema inmune ante la infiltración de sustancias extrañas con alto grado de antigenicidad y bajo grado de biocompatibilidad. La evaluación de la enfermedad se puede hacer de forma cualitativa por evaluación clínica de síntomas inespecíficos de una enfermedad reumática, por evaluación histopatológica o imagenológica, o incluso de una forma cuantitativa por medición directa de reactantes de fase aguda, anticuerpos o autoanticuerpos que enfocan el diagnóstico hacia una enfermedad de causa y efecto inmunológico. Presentamos un caso de enfermedad por modelantes asociado con cáncer de mama.
\end{abstract}

Palabras clave: Enfermedad por modelantes, alogenosis iatrogénica, enfermedad por adyuvante.

\section{ABSTRACT}

Iatrogenic alogenosis or disease by modeling is produced by the indiscriminate use of allogenic or exogenous substances. It is a procedure used since the nineteenth century for aesthetic purposes to improve body contouring. In Mexico, it is a frequent practice; currently represents a serious public health problem. The lack of knowledge of the pathophysiology of iatrogenic alogenosis and the lack of information of patients and medical personnel leads to complications that worsen or exacerbate a picture of silent immunological characteristics. The spectrum of the disease in its local clinical presentation as systemic, is a direct consequence of the activation of the immune system before the infiltration of foreign substances with a high degree of antigenicity and low degree of biocompatibility. The evaluation of the disease can be done qualitatively by clinical evaluation of nonspecific symptoms of a rheumatic disease, by histopathological or imaging evaluation, or even in a quantitative way by direct measurement of acute phase reactants, antibodies or autoantibodies that focus the diagnosis towards a disease of immunological cause and effect. We present a case of disease by modeling associated with breast cancer.

Keywords: Modeling disease, iatrogenic alogenosis, adjuvant disease.
Salazar

E-mail: aantonio.stdra@ gmail.com
Citar como: Pumares CJ, Estrada SLA, Hernández RÁ, Jiménez VX. Enfermedad por modelantes asociado a cáncer de mama. Rev Mex Mastol. 2021; 11 (1): 22-26. https://dx.doi.org/10.35366/99277 


\section{INTRODUCCIÓN}

Las primeras descripciones en el ámbito mundial acerca de las complicaciones originadas por la infiltración de sustancias con fines modelantes datan de hace más de tres siglos, en particular con el uso de petrolato y parafina. Desde la década de 1940, el empleo de silicón líquido en Europa y Estados Unidos, y mezclas oleosas combinadas con silicón, tenían la finalidad de mejorar o cambiar las características físicas de las personas. En los años 70 se dejaron de utilizar por las múltiples manifestaciones patológicas producidas por la introducción de estas sustancias, las cuales dan lugar a la presencia de cuadros clínicos no bien definidos en la literatura. La visión de la enfermedad en su presentación clínica, tanto local como sistémica, es consecuencia directa de la activación del sistema inmune ante la infiltración de sustancias extrañas con alto grado de antigenicidad y bajo grado de biocompatibilidad. La evaluación de la enfermedad se puede hacer de forma cualitativa por evaluación clínica de síntomas inespecíficos de una enfermedad reumática, por evaluación histopatológica o imagenológica, o incluso de una forma cuantitativa por medición directa de reactantes de fase aguda, anticuerpos o autoanticuerpos que enfocan el diagnóstico hacia una enfermedad de causa y efecto inmunológico.
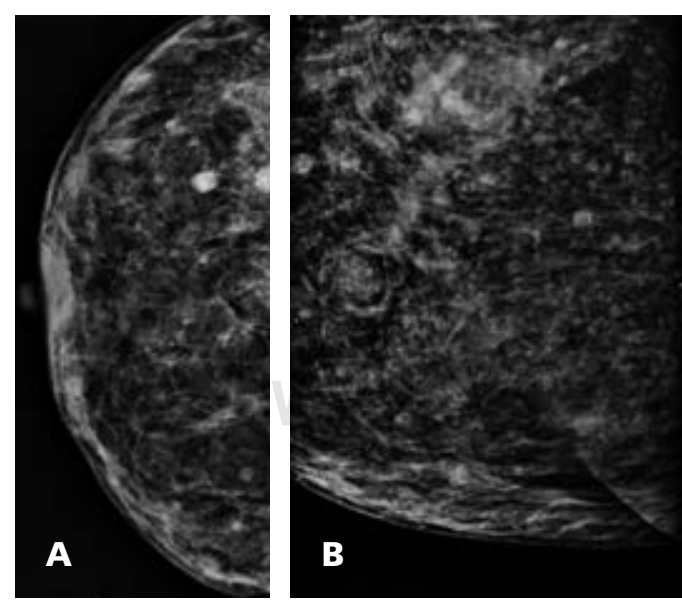

Figura 1: Aumento de la densidad por la presencia de múltiples nódulos hiperdensos. BIRADS 0.

\section{PRESENTACIÓN DEL CASO}

Mujer de 62 años, la cual fue conocida desde el 18 de mayo de 2015, cuenta con los antecedentes de importancia originaria y residente de la Ciudad de México, viuda, ama de casa. Tabaquismo positivo desde los 15 años, dos cajetillas diarias, alcoholismo positivo desde los 25 años de manera semanal hasta llegar a la embriaguez.

Antecedentes heredofamiliares: padre con cáncer de próstata, hermanas con cáncer cervicouterino, tías maternas con diabetes mellitus tipo 2 (DM2), madre con hipertensión arterial sistémica (HAS) y cirrosis hepática con leucemia.

Antecedentes personales: osteoporosis en tratamiento, migraña de 20 años, hipertensión arterial hace cinco años en tratamiento, cardiopatía isquémica crónica dos años de evolución, isquemia cerebral transitoria. Aplicación de silicón en ambas mamas y glúteos hace 20 años.

Inicia con el padecimiento actual en diciembre 2014 con dolor en glándula mamaria izquierda con aumento del tamaño, enrojecimiento, hipertemia y mastalgia.

A la exploración física, mamas asimétricas a expensas de mama izquierda, mama izquierda con fibrosis con tumor en cuadrante superior interno de $3 \times 2 \mathrm{~cm}$, móvil, no adherido a planos profundos. Axila y zonas linfoportadoras negativas. Mama derecha sin lesiones palpables, axila negativa.

La mastografía evidencia aumento de la densidad por la presencia de múltiples nódulos hiperdensos de diversos tamaños con extensión hasta la región retromamaria y cola de Spencer, algunos de los nódulos con calcificación discontinua de sus bordes. BIRADS 0 (Figura 1).

Resonancia magnética de mama con reporte de aumento de la densidad por la presencia de múltiples nódulos hiperdensos (Figura 2).

Se realizaron estudios de extensión (radiografía de tórax), laboratorios prequirúrgicos normales. Biopsia trucut con reporte de carcinoma ductal infiltrante sin patrón específico grado II Scarff-Bloom-Richardson (SBR) 7. 


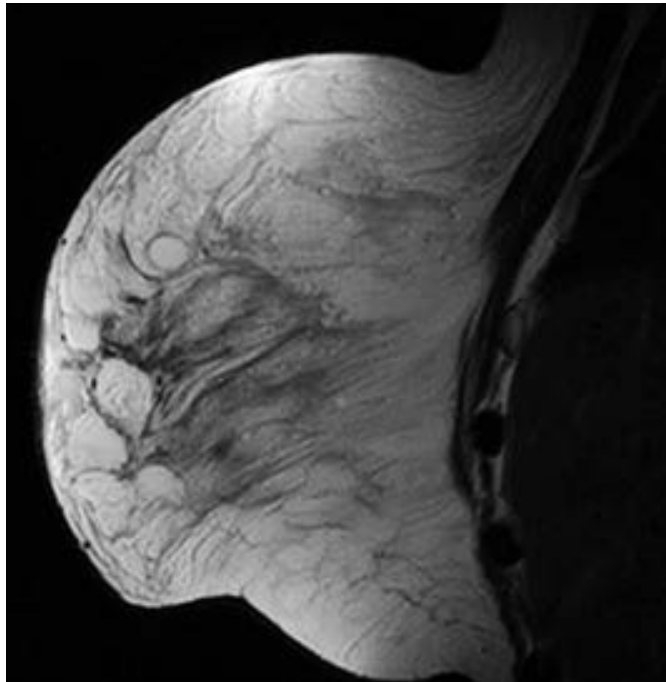

Figura 2: Resonancia magnética de la mama.

Cáncer de mama izquierda EC IIA (T2N0M0)

El 7 de marzo de 2015 se realiza mastectomía radical modificada izquierda con hallazgos: tumor de $2 \times 2 \mathrm{~cm}$ en cuadrante superior interno, ganglios axilares positivos en los tres niveles (Figura 3).

Reporte histopatológico: carcinoma ductal infiltrante sin patrón específico de $8 \mathrm{~mm}$ cuadrante superior izquierdo, permeación linfática intraneoplásica grado II SBR 7 sin necrosis. Con metástasis de un ganglio del nivel I sin ruptura capsular, del nivel II tres ganglios con metástasis y del nivel III dos con hiperplasia linforreticular, carcinoma ductal, mastitis crónica abscedada secundaria a remodelantes.

\section{Inmunohistoquímica}

Receptores de estrógenos 20\%; receptores de progesterona 10\%; Her 2 negativo; P53 negativo. Recibió adyuvancia con quimioterapia con AC secuencial del 10 de abril de 2015 al 03 de septiembre de 2015.

Radioterapia a mama izquierda del 03 de octubre de 2015 al 24 de noviembre de 2015 50 Gy en 25 Fx y del 02 de diciembre de 2015 al 22 de enero de 2016 BOOST 16 Gy en 8 Fx.

El 20 de febrero de 2016 inicia vigilancia por el Servicio de Tumores Mamario, trata- miento hormonal con tamoxifeno. Durante vigilancia inicia con mastalgia derecha, se acompaña con aumento de volumen en región anterior de cuello de mes y medio de evolución, EF nódulo tiroideo derecho de $3 \times 2$ $\mathrm{cm}$, sin adenomegalias. El mismo día se realiza mastectomía simple derecha profiláctica + hemitiroidectomía derecha. Hallazgos: lóbulo tiroideo derecho aumentado de volumen de $6 \times 6 \mathrm{~cm}$ hipervascularizado. Mama péndula con múltiples nódulos. Reporte histopatológico: producto de mastectomía simple derecha: parénquima mamario con inflamación crónica y múltiples quistes, reacción granulomatosa e histiocitos espumosos. Piel y pezones sin alteraciones. Lobectomía tiroidea derecha: adenoma folicular de $3 \mathrm{~cm}$ negativo para malignidad.

\section{DISCUSIÓN}

La asociación de entidades inmunológicas y sustancias exógenas no es desconocida; Miyoshi y colaboradores describieron esta asociación para representar un espectro de enfermedades reumáticas con clínica inespecífica como artralgias, mialgias, alteraciones cognoscitivas, fatiga o incluso fiebre. ${ }^{1,2}$

Los criterios diagnósticos son:

1. Antecedentes de aplicación de sustancias químicas con fines estéticos.

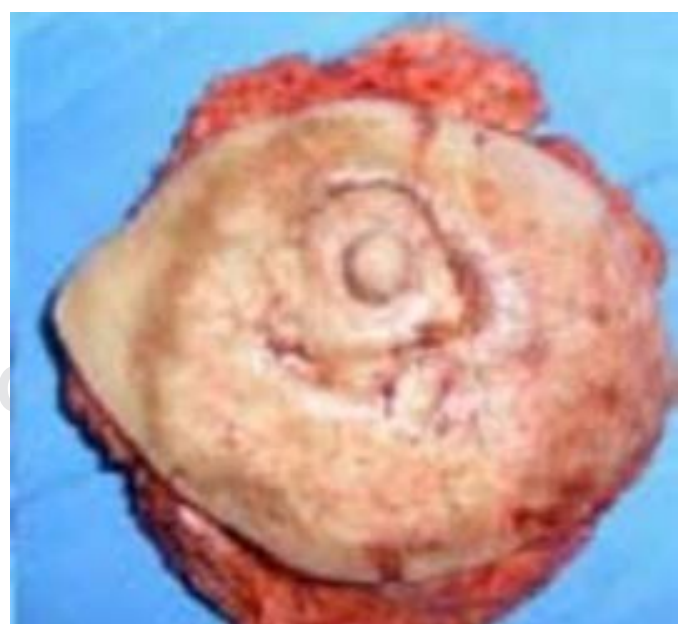

Figura 3: Pieza de mastectomía. 
2. Manifestaciones clínicas de algunas de las enfermedades autoinmunes.

3. Demostración de algún tipo de autoanticuerpo.

4. Evidencia histológica de inflamación crónica y tipo granulomatosa a cuerpo extraño.

5. Ausencia de proceso infeccioso o neoplásico.

6. Mejoría de la sintomatología al retiro de la substancia inyectada o al manejo médico de enfermedad autoinmune. ${ }^{1,2}$

Las manifestaciones clínicas pueden generalizase por la intensa reacción inflamatoria en los tejidos en forma local o a distancia. ${ }^{3}$

Al examen histológico se observan granulomas nodulares con histiocitos epitelioides, células gigantes multinucleadas con cuerpos asteroides y algunos linfocitos, cambios en el tejido con apariencia quística residual y la sustancia alojada dentro de las cavidades quísticas. ${ }^{4}$

Se clasifica por el cuadro clínico y el examen físico de la zona afectada, con cinco grados de severidad. 5,6

Estadio 0: pacientes con cambios locales inespecíficos en zona de infiltración, asintomáticos o sintomáticos, pero sin tumoración palpable o definida.

Estadio I: se palpa tumoración única, piel normal o con cambios discretos de coloración sin deformidad de las subunidades estéticas.

Estadio II: 1) tumoración única o múltiple, signos leves de deformidad de la subunidad con signos de retracción que no compromete la anatomía general; 2) mismas características del punto 1, pero con compromiso muscular y deformidad importante.

Estadio III: tumoración única o múltiple con infiltración de piel y cambios de esclerosis, atrofia, fístula o ulceración y solución de continuidad de la piel con salida espontánea del material.

Estadio IV: compromiso de la unidad estética completa, incluyendo piel hasta profundidad muscular u ósea.

Estadio V: paciente con enfermedad grave con síntomas sistémicos o generalizados que comprometen la calidad de vida y contraindica un procedimiento quirúrgico.

Con base en esta clasificación, se categoriza un posible tratamiento, el cual se muestra a continuación:
Estadio 0: tratamiento médico.

Estadio I: resección limitada del tejido infiltrado y cierre directo.

Estadio II: 1) resección de tumoración subcutánea con remodelación o colocación de implante si se requiere; 2) resección de tumoración subcutánea con cubrimiento con colgajos musculares y/o material aloplástico,

Estadio III: resección amplia de zona indurada con reconstrucción con colgajos musculocutáneos más material aloplástico en caso de ser necesario.

Estadio IV: cirugía paliativa con colgajos musculocutáneos.

Estadio V: tratamiento médico y observación. Sin indicaciones para tratamiento quirúrgico. ${ }^{7,8}$

Se mantendrán en vigilancia los pacientes que fueron candidatos a reconstrucción quirúrgica y resuelto en el segundo nivel cada tres a seis meses.

\section{CONCLUSIONES}

La enfermedad mamaria relacionada con la infiltración de materiales modelantes continúa siendo un problema grave de salud en nuestro país. La necesidad de una evaluación y clasificación adecuada en cada caso, tratamiento médico oportuno y tratamiento quirúrgico acorde con las condiciones de cada paciente aceleran el proceso de detección, manejo sintomático y definitivo. La resonancia magnética nuclear ha demostrado ser el único estudio útil para determinar el grado de afección por el material modelante. Es de gran importancia el manejo multidisciplinario, para evitar el abandono del tratamiento por parte del paciente.

\section{REFERENCIAS}

1. Vera-Lastra O, Medina G, Cruz-Dominguez Mdel P, Ramirez P, Gayosso-Rivera JA, Anduaga-Dominguez $\mathrm{H}$ et al. Human adjuvant disease induced by foreign substances: a new model of ASIA (Shoenfeld's syndrome). Lupus. 2012; 21 (2): 128-135.

2. Juárez A, Durán MA, Rivera AM, Tellez SE, Buitrón $R$, Islas LP. Enfermedad humana por adyuvante en el embarazo. Clin Invest Gin Obst. 2013; 40 (6): 277278. 
3. Zimmermann U, Clerici TJ. The Histological aspects of fillers complications. Semin Cutan Med Surg. 2004; 23: 241-250.

4. Curiel JJ. Mastitis por modelantes. Patología Rev Latinoam. 2010; 48 (3): 216-218.

5. Priego BRB, Cárdenas RJ, Pérez CR, Rincón LR, Torres GB, Haddad JL. Enfermedad humana por modelantes. Análisis de sustancias con espectrometría de resonancia magnética. Cir Plast. 2010; 20 (3): 120-123.

6. Torres GB, Burgos VR, Medrano RG, Priego BRB. Instrumento para evaluar y estadificar el daño producido por la infiltración de sustancias modelantes. Cir Plast. 2010; 20 (3): 105-111.

7. Ortiz-Monasterio F, Trigos I. Management of patients with complications from injections of foreign materials into the breasts. Plast Reconstr Surg. 1972; 50 (1): 42-47.

8. Cabral AR, Alcocer-Varela J, Orozco-Topete R, Reyes E, Fernández-Domínguez L, Alarcón-Segovia D. Clinical, histopathological, immunological and fibroblast studies in 30 patients with subcutaneous injections of modelants including silicone and mineral oils. Rev Invest Clin. 1994; 46 (4): 257-266. 